\title{
Fetal production of growth factors and inflammatory mediators predicts pulmonary hypertension in congenital diaphragmatic hernia
}

\author{
Shannon Fleck', Geoanna Bautista ${ }^{2}$, Sheila M. Keating ${ }^{3}$, Tzong-Hae Lee ${ }^{3}$, Roberta L. Keller ${ }^{1,4}$, Anita J. Moon-Grady ${ }^{1,4}$, \\ Kelly Gonzales ${ }^{2}$, Philip J. Norris ${ }^{3,5,6}$, Michael P. Busch ${ }^{3,6}$, C.J. Kim7, Roberto Romero ${ }^{7}$, Hanmin Lee ${ }^{1,2,5}$, Doug Miniati1 ${ }^{1,2,5}$ \\ and Tippi C. MacKenzie ${ }^{1,2,5}$
}

BACKGROUND: Congenital diaphragmatic hernia (CDH) represents a spectrum of lung hypoplasia, and consequent pulmonary hypertension $(\mathrm{PH})$ is an important cause of postnatal morbidity and mortality. We studied biomarkers at the maternal-fetal interface to understand factors associated with the persistence of $\mathrm{PH}$.

METHODS: Maternal and cord blood samples from fetuses with $\mathrm{CDH}$ and unaffected controls were analyzed using a human 39plex immunoassay kit. Cellular trafficking between the mother and the fetus was quantified using quantitative real-time PCR for nonshared alleles. Biomarker profiles were then correlated with $\mathrm{CDH}$ severity on the basis of the degree of $\mathrm{PH}$.

RESULTS: Cord blood levels of epidermal growth factor, platelet-derived growth factor, and several inflammatory mediators increased significantly as the severity of $\mathrm{CDH}$ increased, whereas maternal levels of growth factors and mediators decreased significantly with $\mathrm{CDH}$ severity. Maternal cells were increased in fetuses with severe $\mathrm{CDH}$ as compared with controls, with elevated levels of the CXC chemokine ligand-10 in patients with the highest trafficking.

CONCLUSION: Patients with CDH demonstrate proinflammatory and chemotactic signals in fetal blood at the time of birth. Because some of these molecules have been implicated in the development of $\mathrm{PH}$, prenatal strategies targeting specific molecular pathways may be useful adjuncts to current fetal therapies.

ongenital diaphragmatic hernia $(\mathrm{CDH})$ remains one of the most challenging congenital anomalies. Although most $\mathrm{CDH}$ patients are now diagnosed before birth, there is a wide spectrum of disease severity, which requires accurate perinatal prognostic indicators for appropriate counseling and management. For patients at the most severe end of the spectrum, in utero tracheal occlusion may promote lung growth and improve postnatal outcomes (1-3). However, in spite of sophisticated fetal and neonatal management, severely affected newborns have a high mortality, and those who survive often have long-term morbidities (4).

Liver position and lung-to-head ratio (LHR) (5) are widely used to measure the degree of pulmonary hypoplasia and predict prognosis in fetuses with $\mathrm{CDH}$. The degree of pulmonary hypertension $(\mathrm{PH})$ is associated with poor outcome in $\mathrm{CDH}$ $(6,7)$ but has been more challenging to predict prenatally. Measurement of pulmonary artery Doppler indexes (8) and maternal hyperoxygenation testing (9) have had some encouraging results but require considerable expertise. A prenatal, minimally invasive biomarker to predict the degree of $\mathrm{PH}$ would greatly benefit patient counseling and management.

In inflammatory disease states associated with $\mathrm{PH}$, such as systemic sclerosis, there is evidence that abnormal levels of growth factors and proinflammatory cytokines lead to vascular remodeling (reviewed in ref. 10). Growth factors signal through tyrosine kinase receptors to induce abnormal proliferation and promote the migration of smooth muscle cells, endothelial cells, and fibroblasts (10). Although the precise mechanisms leading from inflammation to $\mathrm{PH}$ continue to be defined, it is likely that vascular injury resulting from ongoing inflammation leads to pulmonary vascular remodeling (11). The possible contribution of similar pathways in the development of PH in patients with $\mathrm{CDH}$ has not been explored.

We performed an unbiased analysis of growth factors, cytokines, and chemokines in maternal and cord blood to understand the molecular environment contributing to the persistence of $\mathrm{PH}$ in $\mathrm{CDH}$. We also studied the trafficking of cells between the mother and the fetus to determine whether particular cytokines/chemokines secreted in the context of $\mathrm{CDH}$ may alter trafficking. We report that infants with $\mathrm{CDH}$ with persistence of $\mathrm{PH}$ at $2 \mathrm{wk}$ of age have elevated levels of growth factors and proinflammatory cytokines, as well as increased numbers of maternal cells in their cord blood at the time of birth. These findings indicate that molecular signals leading to 
the development of $\mathrm{PH}$ are present before birth and suggest that targeted therapies to block inflammation in utero may be beneficial in patients with severe $\mathrm{CDH}$.

\section{RESULTS}

\section{Patient Demographics}

Maternal and cord blood samples were collected from 19 patients with $\mathrm{CDH}$ and 23 unaffected controls (Table 1). The only significant demographic differences between the groups were that mothers of fetuses with $\mathrm{CDH}$ were more likely to be nulliparous than controls $(P=0.01)$ and were more likely to deliver earlier than controls (median gestational age in weeks (interquartile range): $\mathrm{CDH}: 38.1$ (37.1-38.9) vs. control: 39.0 (38.6-40.0), $P<0.01)$.

\section{CDH Clinical Features}

Seventeen of 19 hernias were left-sided (Table 2). The median LHR was 1.00 (interquartile range: 0.76-1.35) and the median age of LHR measurement was 27 wk (interquartile range: 24 1/7-30 wk). Nine of 14 fetuses with liver herniation into the chest had LHR $<1.0$. There were no survivors among three patients who received extracorporeal membrane oxygenation support. All six neonatal deaths were within the first week of life. The patient with late demise expired at 3 mo of age secondary to severe $\mathrm{PH}$ refractory to medical management.

Three patients with LHR $<1.0$ and liver herniation into the chest underwent fetal endoscopic tracheal occlusion at 27-wk gestation. Of these, one patient was delivered via cesarean section at $38 \mathrm{wk}$ after the balloon was retrieved by a second fetal intervention at $32 \mathrm{wk}$. The two other patients required an ex utero intrapartum treatment procedure (12) at delivery for balloon retrieval at 29 and $36 \mathrm{wk}$, respectively.

\section{Severity Classification}

We applied the CDH severity classification using the degree of $\mathrm{PH}$ measured on echocardiogram performed at $2 \mathrm{wk}$ after birth as described. Under the dichotomous classification, there were six mild and 13 moderate-to-severe patients. Under the threelevel classification, there were six mild, three moderate, and 10 severe patients. All patients with prenatal liver herniation and LHR $<1$ exhibited "severe" $\mathrm{PH}$ at $2 \mathrm{wk}$. We evaluated the relationship of PH to cytokine levels and PCR for microchimerism. Results were similar regardless of which classification scheme was used; two-level results are presented for clarity.

\section{Cytokine Profiles in Cord Blood}

We used a panel of 39 cytokines to analyze whether patients with $\mathrm{CDH}$ have a different cytokine profile in the cord blood plasma as compared with unaffected controls. Cytokine data were available for $17 \mathrm{CDH}$ patients and 22 controls. We found that patients with $\mathrm{CDH}$ overall had elevated levels of epidermal growth factor (EGF), eotaxin, interleukin-3 (IL-3), macrophage inflammatory protein-1 $\beta$ (MIP-1 $\beta$ ), platelet-derived growth factor-AA (PDGF-AA), and IL-1 $\alpha$ in the cord blood as compared with controls (Table 3 ).
We next performed analyses by $\mathrm{CDH}$ disease severity as well as pairwise comparisons. We found that levels of EGF, IL-3, monocyte chemoattractant protein-3 (MCP-3), MIP-1 $\beta$, PDGF-AA, interferon- $\alpha 2$ (IFN- $\alpha 2$ ), and IL- $1 \alpha$ increased significantly across severity groups (control $<$ mild $\mathrm{CDH}<$ moderate-to-severe CDH, $P<0.05$ by Kendall's Tau-c test) (Table 3 and Figure 1a). Further pairwise comparisons suggested that the most consistent differences were between control and mild $\mathrm{CDH}$ as compared with moderate-to-severe $\mathrm{CDH}$ (Table 3). After the Bonferroni adjustment for multiple comparisons, levels of EGF, MIP-1 $\beta$, and IFN- $\alpha 2$ remained significantly elevated in moderate-to-severe $\mathrm{CDH}$ as compared with controls $(P<0.017)$. Of note, a comparison between controls and patients with mild $\mathrm{CDH}$ did not yield any significant differences (Table 3), suggesting that the biomarker profiles seen above are unique to patients with more severe $\mathrm{CDH}$, as measured by the persistence of $\mathrm{PH}$ or demise.

Table 1. Demographics

\begin{tabular}{|c|c|c|c|}
\hline & Control $(n=23)$ & $\mathrm{CDH}(n=19)$ & $P$ value \\
\hline Maternal age (y) & $28(21-34)$ & $31(22-35)$ & 0.72 \\
\hline First pregnancy & $2(8.7 \%)$ & $8(42.1 \%)$ & 0.01 \\
\hline Labor & $14(60.9 \%)$ & $14(73.7 \%)$ & 0.38 \\
\hline \multicolumn{4}{|l|}{ Mode of delivery } \\
\hline Vaginal & $10(43.5 \%)$ & $12(63.2 \%)$ & 0.20 \\
\hline $\begin{array}{l}\text { C-section/ } \\
\text { EXIT }\end{array}$ & $13(56.5 \%)$ & $7(36.8 \%)$ & 0.20 \\
\hline $\begin{array}{l}\text { Gestational age } \\
\text { (weeks) }\end{array}$ & $39.0(38.6-40.0)$ & $38.1(37.1-38.9)$ & 0.001 \\
\hline Male fetus & $11(47.8 \%)$ & $12(63.2 \%)$ & 0.32 \\
\hline Fetal weight (g) & $3,480(3,275-3,850)$ & $3,000(2,000-3,580)^{\mathrm{a}}$ & 0.007 \\
\hline \multicolumn{4}{|c|}{$\begin{array}{l}\text { Data presented as median (interquartile range) or } n \text { (\%); significant } P \text { values are } \\
\text { boldfaced. }\end{array}$} \\
\hline \multicolumn{4}{|c|}{$\mathrm{CDH}$, congenital diaphragmatic hernia; EXIT, ex utero intrapartum treatment procedure. } \\
\hline \multicolumn{4}{|c|}{$\begin{array}{l}\text { aeight in many CDH patients was estimated because patients were too unstable to } \\
\text { weigh. }\end{array}$} \\
\hline
\end{tabular}

Table 2. Patients with congenital diaphragmatic hernia

\begin{tabular}{lc}
\hline CDH characteristics $(n=19)$ & Median (IQR) or $n(\%)$ \\
\hline Left sided & $17(89.5 \%)$ \\
LHR & $1.00(0.76-1.35)$ \\
Liver herniation & $14(73.7 \%)$ \\
Prenatal tracheal occlusion & $3(15.8 \%)$ \\
ECMO & $3(15.8 \%)$ \\
Neonatal demise & $6(31.6 \%)$ \\
Late demise & $1(7.7 \%)$ \\
Repair type $(n=13)$ & \\
Primary & $4(30.8 \%)$ \\
Muscle flap & $5(38.5 \%)$ \\
Patch & $4(30.8 \%)$
\end{tabular}

$\mathrm{CDH}$, congenital diaphragmatic hernia; ECMO, extracorporeal membrane oxygenation; $\mathrm{IQR}$, interquartile range; LHR, lung-to-head ratio. 


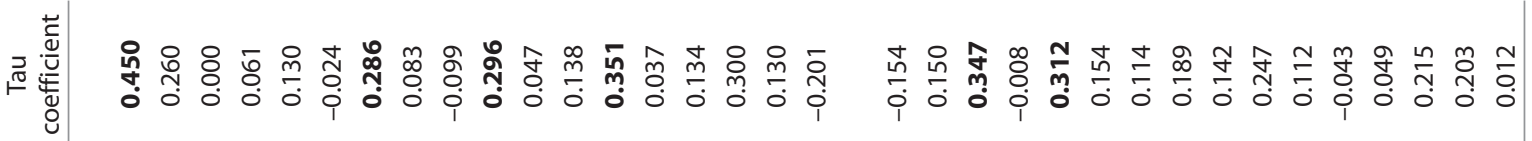

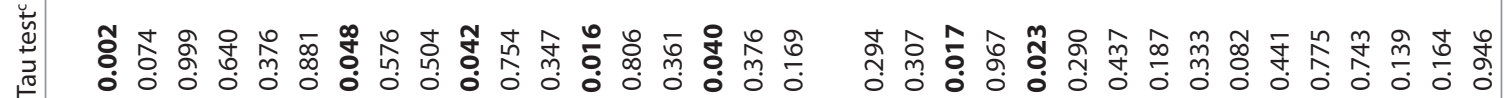

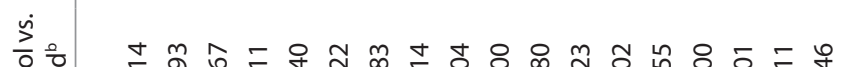

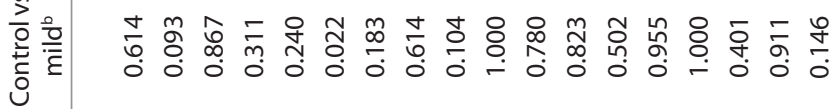

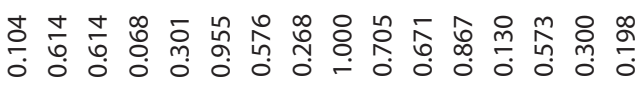

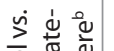

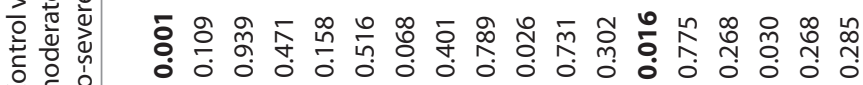

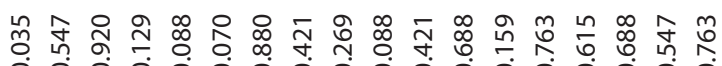

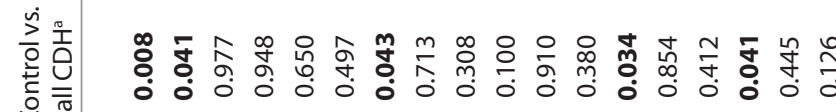

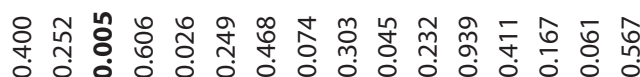

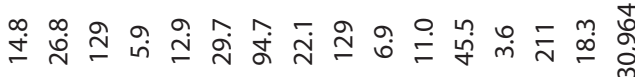

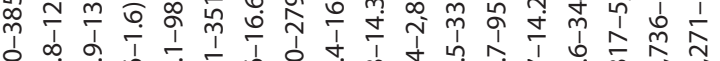

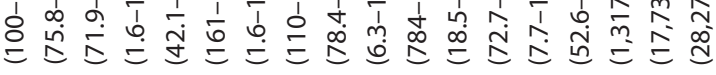

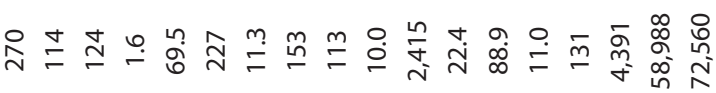

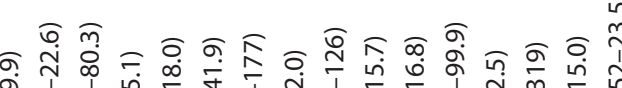

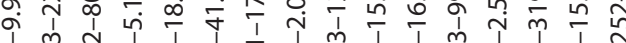
崩 ๙

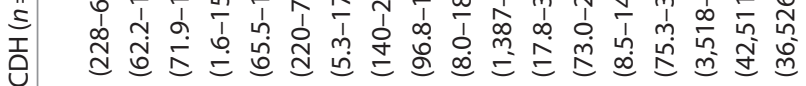

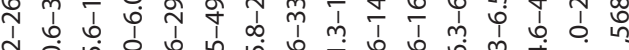
崩 $\bar{\varepsilon}$

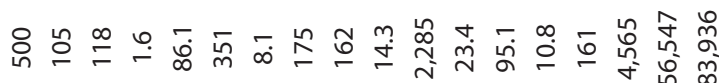

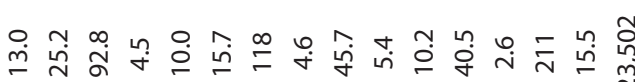

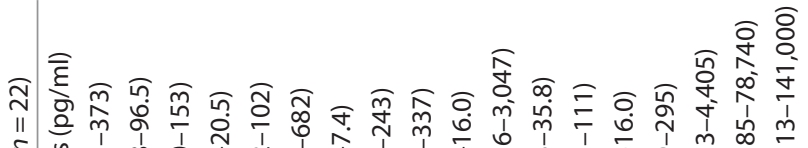

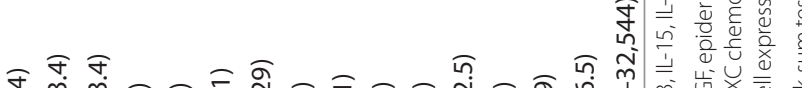

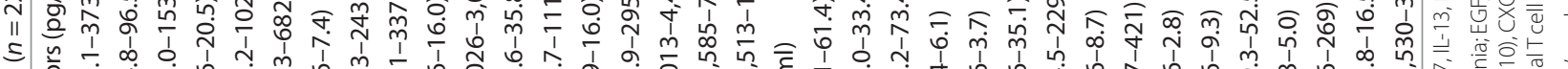

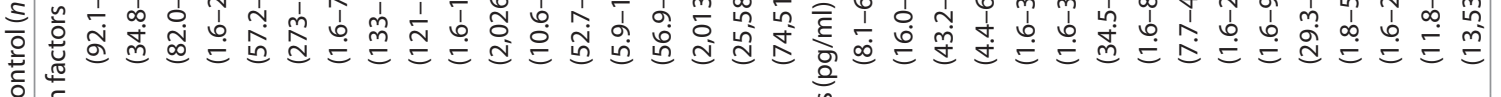
ป

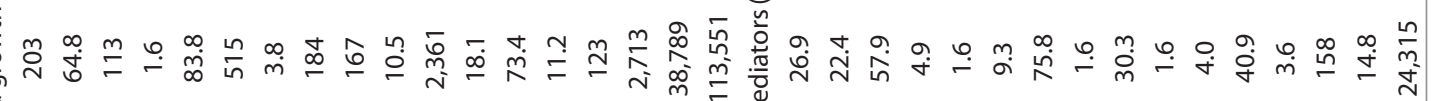
竞贾 㟧

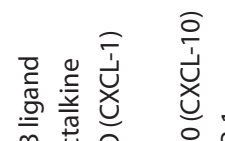




\section{Articles | Fleck et al.}
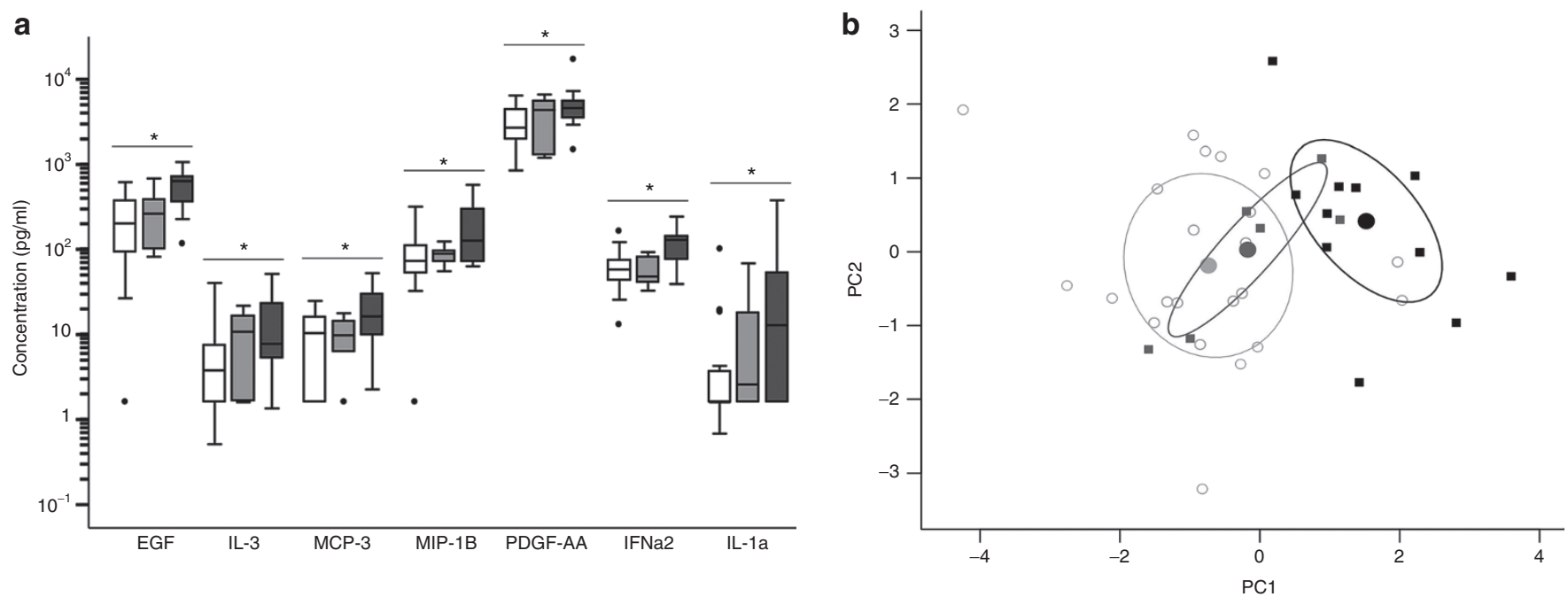

Figure 1. Fetal biomarkers in congenital diaphragmatic hernia (CDH). (a) Levels of growth factors, chemokines, and inflammatory mediators in cord blood of unaffected controls (white bars), patients with mild CDH (light gray bars), and patients with moderate-to-severe CDH (dark gray bars). The horizontal line represents the median for each group. ${ }^{*} P<0.05$ with Kendall's Tau-c test for trend across groups. Control: $n=22$, mild CDH: $n=6$, moderate-tosevere $\mathrm{CDH}: n=11$. EGF, epidermal growth factor; IFN, interferon; IL, interleukin; MCP, monocyte chemoattractant protein; MIP, macrophage inflammatory protein; PDGF, platelet-derived growth factor. (b) Supervised principal component (PC) analysis of biomarkers in cord blood. Biomarkers found to be significantly correlated with severity using Kendall's Tau-c test were entered into a supervised PC analysis. PC1 comprised $35.4 \%$ of the total variability and PC2 comprised 19.3\%. Open circles: control; dark gray squares: mild CDH; black squares: moderate-to-severe CDH. The central point of each cluster is represented by filled circles (light gray: control; dark gray: mild $\mathrm{CDH}$; black: moderate-to-severe $\mathrm{CDH}$ ).

Cytokine levels were also assessed with supervised principal component analysis, which resulted in the reduction of EGF, IL-3, MCP-3, MIP-1 $\beta$, PDGF-AA, IFN- $\alpha 2$, and IL-1 $\alpha$ into two principal components (PCs) that together described $54.7 \%$ of the total variance of the cord blood cytokines. IL-3, MCP-3, MIP- $1 \beta$, IFN- $\alpha 2$, and IL- $1 \alpha$ contributed the most to PC1, whereas EGF and PDGF-AA contributed the most to PC2. The score plot (Figure $\mathbf{1 b}$ ) shows clustering of patients into the three severity groups (control, mild $\mathrm{CDH}$, and moderate-to-severe $\mathrm{CDH}$ ), with separation of control and moderate-to-severe patients, and with mild $\mathrm{CDH}$ found in between the two clusters. Ordinal logistic regression was performed to investigate whether these two principal components were significant predictors of severity. PC1 was significantly associated with higher odds of being classified as more severe (odds ratio $=3.67,95 \%$ confidence interval $=1.74-7.73, P=0.001$ ), whereas PC2 was not (odds ratio $=1.37,95 \%$ confidence inter$\mathrm{val}=0.67-2.76, P=0.381)$. Therefore, an increase in biomarkers related to $\mathrm{PC} 1$ is associated with a greater likelihood of increased $\mathrm{CDH}$ severity.

\section{Cytokine Profiles in Maternal Blood}

In maternal plasma, we first compared all controls to all mothers with $\mathrm{CDH}$ fetuses and found decreased levels of fibroblast growth factor-2 (FGF-2), macrophage-derived chemokine and vascular endothelial growth factor (VEGF) in mothers carrying fetuses with $\mathrm{CDH}$ (Table 4). We also found that maternal levels of FGF-2, macrophage-derived chemokine, and VEGF decreased across groups with increasing severity of $\mathrm{CDH}$ (control > mild $\mathrm{CDH}>$ moderate-to-severe $\mathrm{CDH}$, $P<0.02$ by Kendall's Tau-c test) (Table 4 and Figure 2a). In pairwise comparisons, FGF-2 was significantly decreased in mothers carrying fetuses with moderate-to-severe $\mathrm{CDH}$ as compared with controls $(P=0.014)$.

We performed a supervised principal component analysis for maternal cytokines, which resulted in reduction of FGF-2, macrophage-derived chemokine and VEGF biomarkers into two principal components that explained $90.3 \%$ of the total variance. VEGF contributed the most to PC1, whereas FGF-2 and macrophage-derived chemokine contributed the most to PC2. The three severity groups are less differentiated in the maternal score plot (Figure 2b); however, with ordinal logistic regression, PC1 was found to be significantly associated with a decrease in the odds of carrying a fetus with a moderateto-severe $\mathrm{CDH}$ (odds ratio $=0.41,95 \%$ confidence interval $=$ $0.22-0.75, P=0.001)$.

\section{Maternal-Fetal Cellular Trafficking}

Given the increased levels of several chemokines in the cord blood of patients with $\mathrm{CDH}$, we next examined whether there was increased trafficking of maternal cells into the fetuses in this setting. PCR testing was performed for 18 controls and 19 patients with $\mathrm{CDH}$. Two patients with $\mathrm{CDH}$ and one control had no informative (nonshared) allele for the detection of maternal cells in fetal blood ("maternal microchimerism" $(\mathrm{MMc})$ ), and two patients with $\mathrm{CDH}$ had no informative allele for the detection of fetal cells in maternal blood ("fetal microchimerism" (FMc)). We first analyzed MMc levels by amplifying for nonshared maternal alleles in fetal blood. This analysis showed that there is a range of trafficking in unaffected term infants, as has been reported (13). Among $\mathrm{CDH}$ patients, the median (interquartile range) levels of MMc (expressed as 
赵

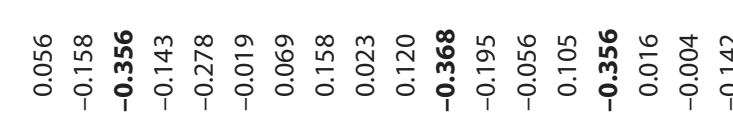

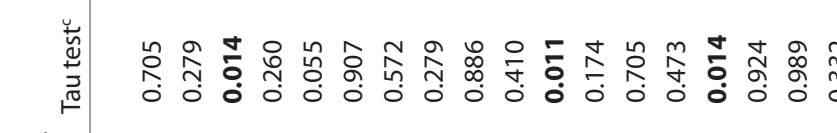

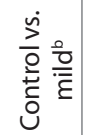

空离尊

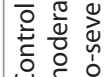

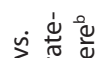

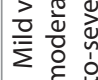

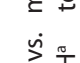

空弯

记

章

离

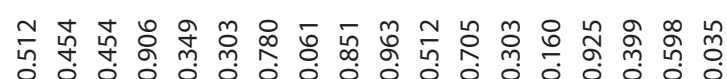

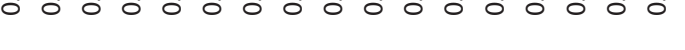

趈

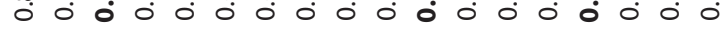

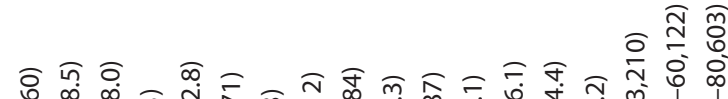
o

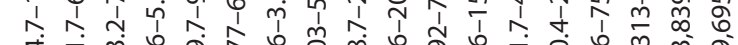

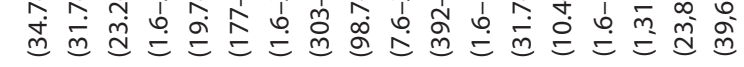

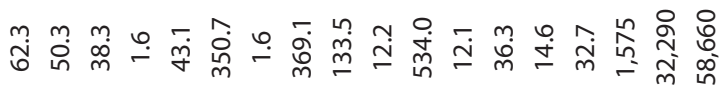

จิ

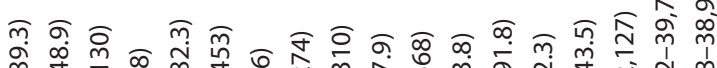

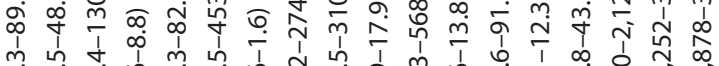

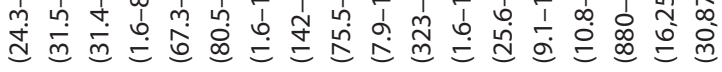
¿ิ่

ล

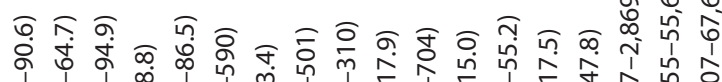

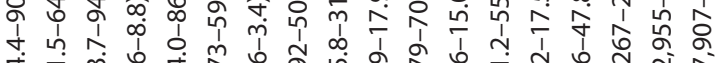

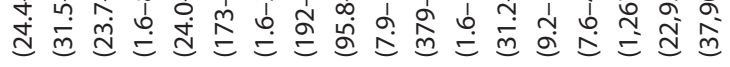

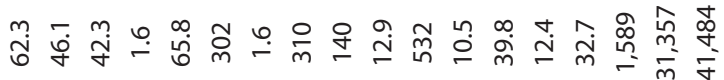

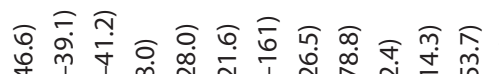

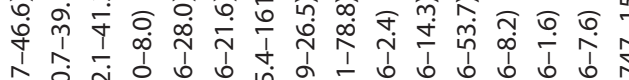

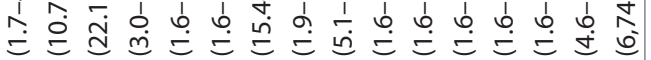
每

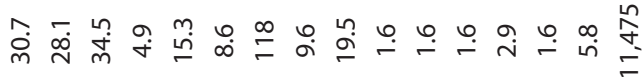

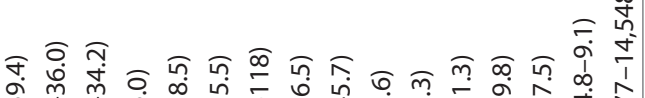

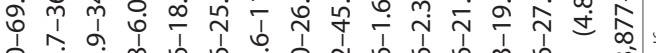

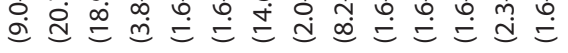

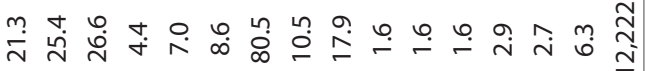
ํํํ 


\section{Articles | Fleck et al.}

a

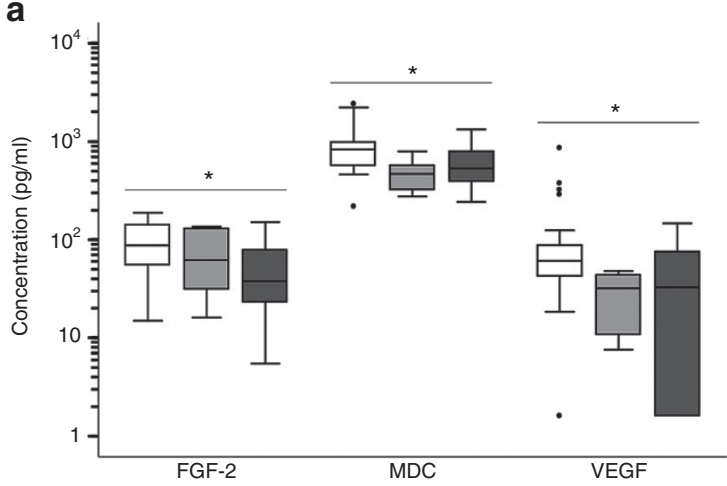

b

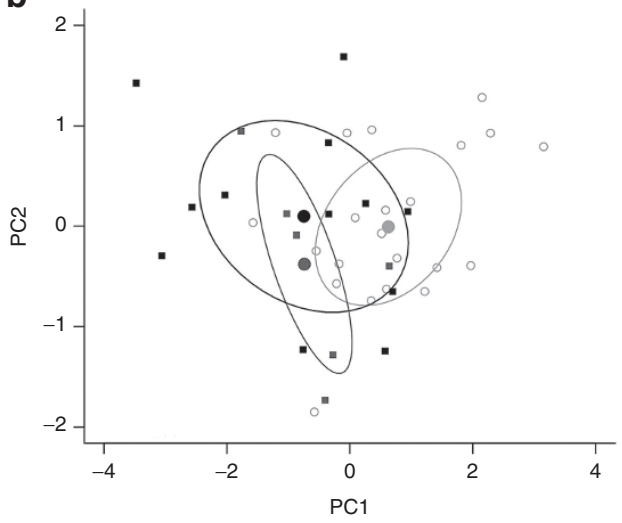

Figure 2. Maternal biomarkers in congenital diaphragmatic hernia (CDH). (a) Levels of growth factors and chemokines in maternal blood in unaffected controls (white bars), patients with mild CDH (light gray bars), and patients with moderate-to-severe CDH (dark gray bars). The horizontal line represents the median for each group. ${ }^{*} P<0.02$ with Kendall's Tau-c test for trend across groups. Control: $n=22$, mild CDH: $n=6$, moderate-to-severe CDH: $n=12$. FGF, fibroblast growth factor; MDC, macrophage-derived chemokine; VEGF, vascular endothelial growth factor. (b) Supervised principal component analysis of biomarkers in maternal blood. Biomarkers found to be significantly correlated with severity using Kendall's Tau-c test were entered into a supervised principal component (PC) analysis. PC1 comprised 35.4\% of the total variability and PC2 comprised 19.3\%. Open circles: control; dark gray squares: mild $\mathrm{CDH}$; black squares: moderate-to-severe $\mathrm{CDH}$. The central point of each cluster is represented by filled circles (light gray: control; dark gray: mild $\mathrm{CDH}$; black: moderate-to-severe $\mathrm{CDH}$ ).
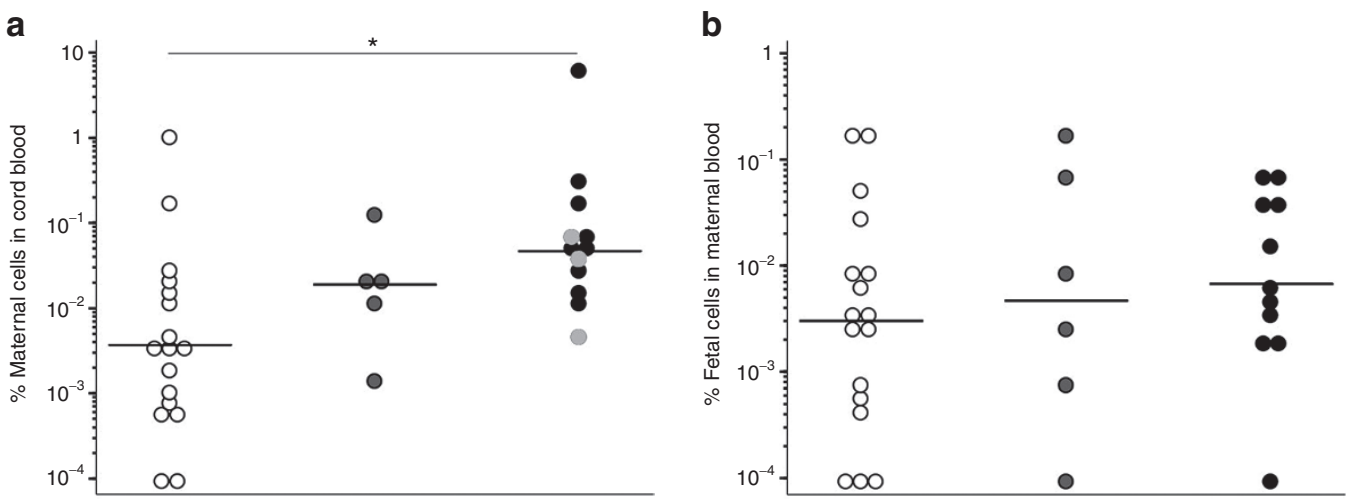

Figure 3. Maternal-fetal cellular trafficking. Percentage of (a) maternal cells in cord blood and of (b) fetal cells in maternal blood in unaffected controls (white circles) and patients with congenital diaphragmatic hernia $(\mathrm{CDH})$ stratified by disease severity (mild CDH: middle column, gray circles; moderateto-severe $\mathrm{CDH}$ : third column, black circles). The horizontal line represents the median for each group. Samples with no microchimerism detected were graphed as $10^{-4}$ (the lower limit of detection) to allow visualization on the log scale. Control $n=17$; mild $\mathrm{CDH} n=5$ in $\mathbf{a}, n=6$ in $\mathbf{b}$; moderate-to-severe $\mathrm{CDH} n=12$ in $\mathbf{a}, n=11$ in $\mathbf{b}$. ${ }^{*} P<0.002$ with Kendall's Tau-c for trend across groups; $P<0.003$ between control and moderate-to-severe CDH by Wilcoxon's rank-sum. Light gray circles in the moderate-to-severe group in a represent patients who underwent tracheal occlusion.

the percentage of maternal cells in cord blood) were significantly higher than in controls (control: $0.004(0.0008-0.015)$ vs. CDH: 0.037 (0.013-0.061), $P<0.004$ by Wilcoxon's ranksum). The levels of MMc tended to increase across severity groups (Kendall's Tau-c coefficient: 0.506, $P<0.002$ ) and were significantly higher in the moderate-to-severe group as compared with controls, whereas there was no difference between mild $\mathrm{CDH}$ and controls (Figure 3a; moderate-to-severe CDH: 0.047 (0.020-0.102), mild CDH: 0.019 (0.011-0.02); $P$ $<0.003$ between moderate-to-severe $\mathrm{CDH}$ and control; $P=$ 0.2 between mild $\mathrm{CDH}$ and control by Wilcoxon's rank-sum). These findings are consistent with the cytokine analysis results in which the main differences were those between controls and patients with moderate-to-severe $\mathrm{CDH}$ and not between controls and patients with mild CDH. The levels of MMc in the three patients who underwent fetal tracheal occlusion were similar to that seen in the other patients with $\mathrm{CDH}(0.004$, 0.037, 0.06; depicted as light gray circles in Figure 3a).

We next quantified levels of FMc by amplifying for nonshared fetal alleles in maternal blood. There were no significant differences between median FMc levels between patients with CDH and unaffected controls (control: 0.003 (0.0006-0.0091) vs. $\mathrm{CDH}$ : $0.007(0.002-0.038), P=0.36$ by Wilcoxon's ranksum). No significant differences were seen when patients with $\mathrm{CDH}$ were stratified into severity groups (Figure $3 \mathbf{b}$; mild CDH: 0.006 (0.0007-0.077), moderate-to-severe CDH: 0.0067 (0.002-0.038), $P=$ not significant with Kendall's Tau-c test).

We next asked whether patients with the highest levels of MMc expressed any cytokines or chemokines that may be implicated in the trafficking of maternal cells across the placenta. We compared the cytokine profiles from cord blood of $\mathrm{CDH}$ patients with high levels of trafficking (MMc level $>80$ th 
percentile, $n=4$ ) to those with lower levels of trafficking (MMc level $\leq 80$ th percentile, $n=13$ ). Consistent with our hypothesis, patients with high MMc had significantly elevated levels of CXC chemokine ligand-10 as compared with those with lower trafficking (726.6 (263.3-1468.8) vs. 173.4 (129.0-271.6), $P=$ 0.027).

\section{DISCUSSION}

This is the first study to directly examine the fetal environment for the presence of biomarkers that may correlate with the persistence of $\mathrm{PH}$ in $\mathrm{CDH}$. We found abnormal levels of several growth factors and cytokines that have been implicated in the development of $\mathrm{PH}$ in other diseases, suggesting that the fetal milieu contains critical molecular signals that lead to vascular changes resulting in $\mathrm{PH}$, even before respiratory effort has begun. Therefore, prenatal therapies to block key events in these molecular pathways may be beneficial in fetuses with $\mathrm{CDH}$.

Our study suggests similarities between the pathophysiology of $\mathrm{PH}$ in $\mathrm{CDH}$ and other diseases. For example, growth factors such as EGF and PDGF have been implicated in the development of $\mathrm{PH}$ in adults (10) and were increased in cord blood of patients with $\mathrm{CDH}$. Although our study does not establish a causal role for these factors in the onset of $\mathrm{PH}$ in $\mathrm{CDH}$, it is interesting to note that blockade of EGF and PDGF has been successful in animal models of PH. For example, EGF blockade may be a useful strategy to treat monocrotaline-induced PH (14). PDGF blockade has also proven beneficial in animal models (15), and may be used clinically in select patients (16).

Inflammatory cytokines implicated in the development of $\mathrm{PH}$ in adults are also increased in fetuses with $\mathrm{CDH}$. For example, IFN- $\alpha$, given to treat hepatitis $C$, reportedly causes irreversible PH in some patients (17), and was increased in our cohort of patients with moderate-to-severe $\mathrm{CDH}$. Increased levels of IL-1, IL-6, and tumor necrosis factor- $\alpha$ have been described in patients with primary $\mathrm{PH}(18,19)$, and our study indicated some increases in these cytokines with severe $\mathrm{CDH}$.

Conversely, several cytokines and/or chemokines described in the pathogenesis of $\mathrm{PH}$ in other disease settings, such as fractalkine (20), were not increased in our CDH patients. In addition, we did not find elevated levels of anti-inflammatory cytokines such as IL-10 in patients with $\mathrm{CDH}$, as has been reported in adults with idiopathic PH (19). This discrepancy may be because our assay could not detect small changes in IL-10 because the baseline levels are low, or because of the inability of the fetus to compensate, unlike adults with PH.

We also analyzed maternal plasma in an effort to define a biomarker that may be followed noninvasively and found significantly decreased levels of FGF-2 in mothers of fetuses with moderate-to-severe CDH. FGF-2 has been implicated in the pathogenesis of $\mathrm{PH}$, and its blockade may ameliorate disease in an experimental model (21); we speculate that our findings may indicate a compensatory mechanism.

A recent study examined the levels of various cytokines in the blood of neonates with $\mathrm{CDH}$, drawn shortly after birth and longitudinally over $4 \mathrm{~d}$ (22). The authors detected elevated levels of several proinflammatory cytokines in infants with $\mathrm{CDH}$ as compared with unaffected controls, although blood from patients with $\mathrm{CDH}$ was drawn after the initial resuscitation, whereas control blood was obtained from the umbilical cord. The authors also compared patients who did or did not require extracorporeal membrane oxygenation and found that levels of IL-8, IL-10, and MIP- $1 \alpha$ were increased in neonates with more severe $\mathrm{CDH}$. Our study adds to these observations by directly comparing both maternal and cord blood levels before any respiratory effort or mechanical ventilation, and by including other analytes known to be involved in PH, such as EGF, PDGF, and FGF. In addition, we have directly correlated cytokine levels with the degree of $\mathrm{PH}$ measured on echocardiogram to show that the differences between controls and patients with $\mathrm{CDH}$ are most pronounced in patients with moderate-to-severe $\mathrm{PH}$ and that patients with mild $\mathrm{CDH}$ exhibit little inflammation. Cellular trafficking between the mother and the fetus has been described in normal pregnancies $(23,24)$, and may be a mechanism for the induction of maternal-fetal tolerance (25). The observation that patients with more severe $\mathrm{CDH}$ have higher levels of MMc as compared with controls is intriguing and suggests that molecular signals present in some $\mathrm{CDH}$ patients may lead to the recruitment of maternal cells or to the proliferation of maternal cells that have already crossed into the fetus. The finding of increased levels of CXC chemokine ligand-10 in patients with the highest levels of trafficking supports this hypothesis. It has been reported that endothelial progenitor cells are recruited from the bone marrow to the pulmonary vasculature in animal models of PH (26), and similar signals may lead to the recruitment of maternal cells in patients with $\mathrm{CDH}$. Of note, we did not find elevated MMc after fetal intervention, contrary to what we have seen in our mouse model of fetal intervention (27) or after open fetal surgery for spina bifida (28). It is possible that the minimally invasive nature of the current approach to tracheal occlusion does not lead to increased trafficking, or signals leading to increased trafficking are different between patients with spina bifida and those with $\mathrm{CDH}$.

The main strengths of our analysis are the study of both maternal and cord blood in patients with $\mathrm{CDH}$ and our unbiased approach of testing for multiple cytokines without predicting which ones may be changed on the basis of published data. We are also aware of several weaknesses in our study. The mean gestational age in patients with $\mathrm{CDH}$ was lower than that for our controls, which may be one confounding factor in our analysis. The ideal control group would be age-matched unaffected controls, but patients born at $36 \mathrm{wk}$ usually have other abnormalities prompting delivery, such as infection or maternal comorbidity, and therefore would not be an appropriate comparison group. In addition, our hospital referral patterns led to a $\mathrm{CDH}$ cohort with fewer patients with mild $\mathrm{CDH}$. Finally, longitudinal assessments of biomarkers in maternal blood or amniotic fluid may lead to the identification of prognostic factors before $24 \mathrm{wk}$, when such information may inform the course of prenatal care, and such a study may be designed in the future. 
Tracheal occlusion is currently the mainstay of fetal intervention, but is likely to be more effective for treating pulmonary hypoplasia than for treating $\mathrm{PH}$ (29). Our finding that prenatal inflammatory signals correlate with the severity of postnatal $\mathrm{PH}$ suggests that prenatal strategies to block molecular pathways may be beneficial in severe $\mathrm{CDH}$, similar to the targeted therapies currently being developed for $\mathrm{PH}$ in other settings (30). Animal studies of prenatal medical therapies to address $\mathrm{PH}$ in $\mathrm{CDH}$ have shown encouraging results. For example, prenatal steroids improved pulmonary vascular remodeling in the lamb model of $\mathrm{CDH}$ (31), and antenatal sildenafil had beneficial effects on lung microvascular development in a rat model of $\mathrm{CDH}$ (32). Our results, in conjunction with other reports describing increased EGF in patients with $\mathrm{CDH}$ (33), point to EGF inhibition as another potential therapeutic target. PDGF was recently reported to reduce pulmonary vascular remodeling in a rat model of $\mathrm{CDH}$ (34) and was used clinically in one patient with $\mathrm{CDH}$ (35). However, potential toxicities such as impairment in alveolar development owing to inhibition of PDGF receptor signaling must be considered.

This is the first study to demonstrate an association between maternal and cord blood biomarker profiles and the persistence of $\mathrm{PH}$ in $\mathrm{CDH}$. Our results lend insight into the fetal onset of $\mathrm{PH}$ and suggest that prenatal therapies to block particular molecular pathways may be useful in prenatally diagnosed $\mathrm{CDH}$.

\section{METHODS \\ Subjects}

Mothers carrying unaffected fetuses or those with $\mathrm{CDH}$ were prospectively enrolled (August 2009-June 2011). Inclusion and exclusion criteria for all healthy control patients were term pregnancies without preterm labor, preeclampsia, or fetal congenital anomalies. All consenting patients with fetal $\mathrm{CDH}$ were prospectively enrolled. Matched maternal and cord blood samples were obtained at birth and only patients with a matched pair of samples were included in the study. Written informed consent was obtained under University of California, San Francisco Institutional Review Board approval (10-00350). All patients with $\mathrm{CDH}$ were evaluated by fetal ultrasound and echocardiogram. Fetal tracheal occlusion was performed in three patients with severe $\mathrm{CDH}$ (liver herniated into the thorax and LHR $<1.0)$. All neonates with $\mathrm{CDH}$ were managed as previously described (7). Surgical repair was performed after the patient was stabilized and repair type was dependent on the size of the diaphragm defect and surgeon preference.

\section{Postnatal Classification of Severity in Infants With CDH}

We have previously shown that the severity of $\mathrm{PH}$ at $2 \mathrm{wk}$ of age (on the basis of an estimate of pulmonary artery pressure $\left(\mathrm{P}_{\mathrm{PA}}\right)$ from the echocardiogram) is associated with poor neonatal outcome (7). A single cardiologist (A.J.M.-G.), blinded to patient condition, reviewed echocardiograms performed at $2 \mathrm{wk}$ after birth and classified $\mathrm{P}_{\mathrm{PA}}$ relative to systemic blood pressure (7). In a three-level classification system, infants were classified as "mild $\mathrm{CDH}$ " if there was no or mild

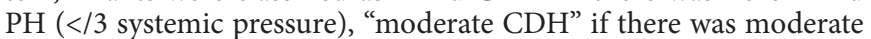
$\mathrm{PH}(\geq 2 / 3$ systemic pressure) and "severe $\mathrm{CDH}$ " if there was severe $\mathrm{PH}$ (systemic-to-suprasystemic pressure) or demise before $2 \mathrm{wk}$. Alternatively, in a dichotomous classification system, infants were classified as "mild CDH" vs. "moderate-to-severe CDH" ( $\geq 2 / 3$ systemic pressure or demise).

\section{Sample Processing}

Cord blood samples were collected at the time of birth and maternal blood was collected within $24 \mathrm{~h}$ of delivery and processed within $36 \mathrm{~h}$.
Whole blood and plasma were stored separately for the analysis of cellular trafficking and cytokines, respectively. Blood obtained from patients delivering in Detroit (11/23 controls) was shipped on ice on the day of delivery by overnight mail and processed immediately upon arrival, such that the timing and method of processing of all samples was identical.

\section{Cytokine Assay}

Cytokine profiles in the maternal and cord blood plasma samples were assayed using the standard-sensitivity Millipex Map kit (Millipore, Billerica, MA) as previously reported (36). Samples were acquired and analyzed on a Labscan 100 analyzer (Luminex, Austin, TX) using BioPlex manager 6.0 software (Bio-Rad, Hercules, CA). Standard curves were run in duplicate wells and each run included internal controls. Cytokines were excluded from the tables and figures if their levels were below $10 \mathrm{pg} / \mathrm{ml}$ for at least $80 \%$ of both maternal and cord blood samples.

\section{Quantitative PCR}

MMc and FMc were quantified from whole blood by researchers blinded to patient groups using a quantitative reverse transcriptionPCR assay to amplify nonshared human leukocyte antigen-DR or insertion-deletion alleles between the mother and the fetus (37). This assay has a lower limit of detection between 0.001 and $0.0001 \%$ (37) and has been used previously to quantify maternal blood in fetal samples $(25,28)$. Microchimerism levels in two CDH patients who underwent fetal intervention and seven controls have been reported as control data in the context of an analysis of the effects of open fetal surgery on trafficking (28).

\section{Statistical Analysis}

Wilcoxon's rank-sum test with Bonferroni-adjusted $P$ values for multiple comparisons was used where appropriate. $\chi^{2}$ tests were used to assess the differences in proportions between groups. Kendall's Tau-c statistic was calculated to test the magnitude and direction of increases or decreases in cytokine levels or microchimerism across groups. Cytokine levels were also assessed by supervised principal component analysis, which uses only a subset of cytokines most associated with the severity of outcome $(P<0.05$ by Kendall's Tau-c) for reducing the dimensionality of the data. The principal components identified were then entered as predictors of severity in an ordinal logistic regression model. A $P$ value $<0.05$ was considered statistically significant. All statistical analyses were performed using Stata version 12 (Stata, College Station, TX) or R software (Vienna, Austria).

\section{ACKNOWLEDGMENTS}

We thank the physicians and nurses at the University of California, San Francisco Labor and Delivery Unit and the Fetal Treatment Center for their assistance with sample collection; Qizhi Tang, Peter Oishi, and Jeff Fineman for helpful discussions; and our patients for their gracious participation in this research project.

\section{STATEMENT OF FINANCIAL SUPPORT}

This work was supported by a March of Dimes Basil O'Connor Award, White Plains, NY (to T.C.M.), and grants from the University of California, San Francisco Resource Allocation Program (to T.C.M.), the California Institute for Regeneration Medicine (to T.C.M.), the US National Heart, Lung, and Blood Institute (R01-HL-088388, to M.P.B.), and the Pulmonary Hypertension Association, Silver Spring, MD (to R.L.K.).

\section{REFERENCES}

1. Deprest JA, Gratacos E, Nicolaides K, et al. Changing perspectives on the perinatal management of isolated congenital diaphragmatic hernia in Europe. Clin Perinatol 2009;36:329-47, ix.

2. Jelin E, Lee $\mathrm{H}$. Tracheal occlusion for fetal congenital diaphragmatic hernia: the US experience. Clin Perinatol 2009;36:349-61, ix.

3. Ruano R, Duarte SA, Pimenta EJ, et al. Comparison between fetal endoscopic tracheal occlusion using a 1.0-mm fetoscope and prenatal expectant management in severe congenital diaphragmatic hernia. Fetal Diagn Ther 2011;29:64-70. 
4. Jancelewicz T, Vu LT, Keller RL, et al. Outcomes of multigestational pregnancies affected by congenital diaphragmatic hernia. J Pediatr Surg 2010;45:1753-8.

5. Hedrick HL, Danzer E, Merchant A, et al. Liver position and lung-to-head ratio for prediction of extracorporeal membrane oxygenation and survival in isolated left congenital diaphragmatic hernia. Am J Obstet Gynecol 2007;197:422.e1-4.

6. Dillon PW, Cilley RE, Mauger D, Zachary C, Meier A. The relationship of pulmonary artery pressure and survival in congenital diaphragmatic hernia. J Pediatr Surg 2004;39:307-12; discussion 307-12.

7. Keller RL, Tacy TA, Hendricks-Munoz K, et al. Congenital diaphragmatic hernia: endothelin-1, pulmonary hypertension, and disease severity. Am J Respir Crit Care Med 2010;182:555-61.

8. Fuke S, Kanzaki T, Mu J, et al. Antenatal prediction of pulmonary hypoplasia by acceleration time/ejection time ratio of fetal pulmonary arteries by Doppler blood flow velocimetry. Am J Obstet Gynecol 2003;188:228-33.

9. Broth RE, Wood DC, Rasanen J, et al. Prenatal prediction of lethal pulmonary hypoplasia: the hyperoxygenation test for pulmonary artery reactivity. Am J Obstet Gynecol 2002;187:940-5.

10. Schermuly RT, Ghofrani HA, Wilkins MR, Grimminger F. Mechanisms of disease: pulmonary arterial hypertension. Nat Rev Cardiol 2011;8:443-55.

11. Hassoun PM, Mouthon L, Barberà JA, et al. Inflammation, growth factors, and pulmonary vascular remodeling. J Am Coll Cardiol 2009;54:Suppl 1:S10-9.

12. Mychaliska GB, Rice HE, Tarantal AF, et al. In utero hematopoietic stem cell transplants prolong survival of postnatal kidney transplantation in monkeys. J Pediatr Surg 1997;32:976-81.

13. Hall JM, Lingenfelter P, Adams SL, Lasser D, Hansen JA, Bean MA. Detection of maternal cells in human umbilical cord blood using fluorescence in situ hybridization. Blood 1995;86:2829-32.

14. Merklinger SL, Jones PL, Martinez EC, Rabinovitch M. Epidermal growth factor receptor blockade mediates smooth muscle cell apoptosis and improves survival in rats with pulmonary hypertension. Circulation 2005;112:423-31.

15. Schermuly RT, Dony E, Ghofrani HA, et al. Reversal of experimental pulmonary hypertension by PDGF inhibition. J Clin Invest 2005; 115:2811-21.

16. Grimminger F, Schermuly RT. PDGF receptor and its antagonists: role in treatment of PAH. Adv Exp Med Biol 2010;661:435-46.

17. Dhillon S, Kaker A, Dosanjh A, Japra D, Vanthiel DH. Irreversible pulmonary hypertension associated with the use of interferon alpha for chronic hepatitis C. Dig Dis Sci 2010;55:1785-90.

18. Humbert M, Monti G, Brenot F, et al. Increased interleukin-1 and interleukin-6 serum concentrations in severe primary pulmonary hypertension. Am J Respir Crit Care Med 1995;151:1628-31.

19. Soon E, Holmes AM, Treacy CM, et al. Elevated levels of inflammatory cytokines predict survival in idiopathic and familial pulmonary arterial hypertension. Circulation 2010;122:920-7.

20. Balabanian K, Foussat A, Dorfmüller P, et al. CX(3)C chemokine fractalkine in pulmonary arterial hypertension. Am J Respir Crit Care Med 2002;165:1419-25.
21. Izikki M, Guignabert C, Fadel E, et al. Endothelial-derived FGF2 contributes to the progression of pulmonary hypertension in humans and rodents. J Clin Invest 2009;119:512-23.

22. Schaible T, Veit M, Tautz J, et al. Serum cytokine levels in neonates with congenital diaphragmatic hernia. Klin Padiatr 2011;223:414-8.

23. Bianchi DW, Zickwolf GK, Weil GJ, Sylvester S, DeMaria MA. Male fetal progenitor cells persist in maternal blood for as long as 27 years postpartum. Proc Natl Acad Sci USA 1996;93:705-8.

24. Maloney S, Smith A, Furst DE, et al. Microchimerism of maternal origin persists into adult life. J Clin Invest 1999;104:41-7.

25. Mold JE, Michaëlsson J, Burt TD, et al. Maternal alloantigens promote the development of tolerogenic fetal regulatory $\mathrm{T}$ cells in utero. Science 2008;322:1562-5.

26. Hayashida K, Fujita J, Miyake Y, et al. Bone marrow-derived cells contribute to pulmonary vascular remodeling in hypoxia-induced pulmonary hypertension. Chest 2005;127:1793-8.

27. Nijagal A, Wegorzewska M, Jarvis E, Le T, Tang Q, MacKenzie TC. Maternal $\mathrm{T}$ cells limit engraftment after in utero hematopoietic cell transplantation in mice. J Clin Invest 2011;121:582-92.

28. Saadai P, Lee TH, Bautista G, et al. Alterations in maternal-fetal cellular trafficking after fetal surgery. J Pediatr Surg 2012;47:1089-94.

29. Danzer E, Davey MG, Kreiger PA, et al. Fetal tracheal occlusion for severe congenital diaphragmatic hernia in humans: a morphometric study of lung parenchyma and muscularization of pulmonary arterioles. J Pediatr Surg 2008;43:1767-75.

30. O'Callaghan DS, Savale L, Montani D, et al. Treatment of pulmonary arterial hypertension with targeted therapies. Nat Rev Cardiol 2011;8:526-38.

31. Davey M, Shegu S, Danzer E, et al. Pulmonary arteriole muscularization in lambs with diaphragmatic hernia after combined tracheal occlusion/glucocorticoid therapy. Am J Obstet Gynecol 2007;197:381.e1-7.

32. Luong C, Rey-Perra J, Vadivel A, et al. Antenatal sildenafil treatment attenuates pulmonary hypertension in experimental congenital diaphragmatic hernia. Circulation 2011;123:2120-31.

33. Guarino N, Solari V, Shima H, Puri P. Upregulated expression of EGF and TGF-alpha in the proximal respiratory epithelium in the human hypoplastic lung in congenital diaphragmatic hernia. Pediatr Surg Int 2004;19: 755-9.

34. Chang YT, Ringman Uggla A, Osterholm C, et al. Antenatal imatinib treatment reduces pulmonary vascular remodeling in a rat model of congenital diaphragmatic hernia. Am J Physiol Lung Cell Mol Physiol 2012;302:L1159-66.

35. Frenckner B, Broomé M, Lindström M, Radell P. Platelet-derived growth factor inhibition-a new treatment of pulmonary hypertension in congenital diaphragmatic hernia? J Pediatr Surg 2008;43:1928-31.

36. Keating SM, Golub ET, Nowicki M, et al.; Women's Interagency HIV Study. The effect of HIV infection and HAART on inflammatory biomarkers in a population-based cohort of women. AIDS 2011;25:1823-32.

37. Lee TH, Chafets DM, Reed W, et al. Enhanced ascertainment of microchimerism with real-time quantitative polymerase chain reaction amplification of insertion-deletion polymorphisms. Transfusion 2006;46: $1870-8$. 